\title{
Association of genetic polymorphisms in the RET-protooncogene and NRG1 with Hirschsprung disease in Thai patients
}

\author{
Theerawut Phusantisampan ${ }^{1}$, Surasak Sangkhathat ${ }^{2}$, Amornrat Phongdara ${ }^{1}$, Piyawan Chiengkriwate ${ }^{2}$, \\ Sakda Patrapinyokul ${ }^{2}$ and Surakameth Mahasirimongkol ${ }^{3}$
}

Hirschsprung disease (HSCR) is a congenital developmental defect of the enteric nervous system known to be associated with the RET-protooncogene and other candidates. Recently, a genome-wide association study has added NRG1, a regulator of the development of the enteric ganglia precursors, as a new candidate gene. The aim of this study is to validate the association of the RET-protooncogene and the NRG1 in HSCR in Thai patients. The study used TaqMan single-nucleotide polymorphism (SNP) genotyping and PCR-restriction fragment length polymorphism for genotyping of $10 \mathrm{SNPs}$ within the RET-protooncogene and four SNPs within the NRG1, in 68 Thai sporadic HSCR cases and 120 ethnic-matched controls. On univariate disease association analysis, 9 of 10 RET-protooncogene SNPs and all four NRG1 SNPs showed an association with HSCR. The rs2435357 (RET-protooncogene) and rs2439305 (NRG1) showed the strongest associations with the disease at $P$-values of 8.17E-09 (odds ratio $(\mathrm{OR})=6.43,95 \%$ confidence intervals $(\mathrm{CI})=3.33-12.40)$ and $6.94 \mathrm{E}-03(\mathrm{OR}=3.28,95 \% \mathrm{Cl}=1.28-8.38)$, respectively. The RET-protooncogene rs2435357 (TT genotype) in combination with the NRG1 rs2439305 (GG genotype) was strongly associated with an increased risk of HSCR with a $P$-value of $1.99 \mathrm{E}-04(\mathrm{OR}=20.34,95 \% \mathrm{Cl}$; 2.54-162.78) when compared with a single SNP of the RET-protooncogene or NRG1. Genetic variation of the RET-protooncogene and NRG1 is involved in the risk of HSCR development in the Thai population. Moreover, the study also detected a combined effect of SNPs by SNP-SNP interaction, which may help in predicting HSCR risk.

Journal of Human Genetics (2012) 57, 286-293; doi:10.1038/jhg.2012.18; published online 1 March 2012

Keywords: congenital megacolon; NRG1; RET-protooncogene

\section{INTRODUCTION}

Hirschsprung disease (HSCR) or congenital megacolon (OMIM 142643), one of the complex genetic disorders, is characterized by functional colonic obstruction secondary to an absence of ganglion cells in the distal part of the gastrointestinal tract. ${ }^{1}$ The incidence of this disease is generally 1 per 5000 of live births, with males about 3.57.8 times more likely to be affected than females. ${ }^{2,3}$ There are also differences among races, with a higher incidence in Asians at 2.8 per 10000 of live births. ${ }^{4}$ The incidence of HSCR in Thailand has never been studied, but it is believed to be more prevalent in the southern part of the country. ${ }^{5}$

The etiology of HSCR is multifactorial, involving multiple genetic and environmental factors. ${ }^{6}$ To date, at least 11 genes have been identified as susceptibility loci in HSCR: RET-protooncogene, GDNF, NTN, EDNRB, EDN3, SOX10, ECE-1, ZFHX1B, РHOX2B,
KIAA12794,6 and the most recently found, NRG1, identified by a genome-wide association study (GWAS). ${ }^{7,8}$

The RET-protooncogene encodes a tyrosine kinase receptor and belongs to a signaling pathway, which has essential roles in the development of the enteric nervous system. ${ }^{9-11}$ Recent studies have suggested the RET-protooncogene as a major locus involved in HSCR pathogenesis. ${ }^{11-13}$ Single-nucleotide polymorphisms (SNPs) of the RET-protooncogene have been demonstrated to have an association with HSCR in various studies in Asians, ${ }^{12-16}$ the majority of these studies were conducted in East Asian populations.

NRG1 was identified as a novel HSCR susceptibility gene in a recent GWAS report, having roles in neuronal development and migration, synaptogenesis and neuronal transmission by binding to ErbB receptors. $^{7}$ In this study, we selected two genes on the basis that the RETprotooncogene is already known to be associated with the disease and

${ }^{1}$ Center for Genomics and Bioinformatics Research, Faculty of Science, Prince of Songkla University, Hat Yai, Songkhla, Thailand; ${ }^{2}$ Department of Surgery, Faculty of Medicine, Pediatric Surgery Unit and Tumor Biology Research Unit, Prince of Songkla University, Hat Yai, Thailand and ${ }^{3}$ Medical Genetics Section, National Institute of Health, Department of Medical Sciences, Ministry of Public Health, Nonthaburi, Thailand

Correspondence: Dr S Sangkhathat, Department of Surgery, Faculty of Medicine, Pediatric Surgery Unit and Tumor Biology Research Unit, Prince of Songkla University, Hat Yai, Songkhla 90110, Thailand.

E-mail: surasak.sa@psu.ac.th

Received 22 October 2011; revised 22 January 2012; accepted 31 January 2012; published online 1 March 2012 
NRG1 as a novel gene that it would be of interest to have validated in our population. In addition, both genes have functions in neuronal development, where they possibly interact with each other. ${ }^{4,7}$ However, an association in terms of genetic epidemiology between NRG1 and RET-protooncogene has not been previously reported. In this study, we aimed to investigate any potential associations between SNPs on the RET-protooncogene and NRG1 genes, and HSCR. A secondary objective was to study the SNP-SNP interactions between the two genes, with an aim to develop a useful marker for disease prediction.

\section{MATERIALS AND METHODS}

\section{Study subjects}

The study was approved by the Research Ethics Committee of the Faculty of Medicine, Prince of Songkla University. A total of 68 patients, ages $0-15$ years, with histologically proven HSCR, who underwent surgery in our institute during the years 2003-2009, were recruited into the study following informed consent. The disease severity of each patient was categorized by the extent of aganglionosis. Long-segment HSCR was defined as aganglionosis that extended proximal to the recto-sigmoid region including total colonic aganglionosis, whereas the remaining cases were grouped as short-segment HSCR. The controls were 120 ethnic-matched healthy volunteers, aged $>15$ years, with no history of chronic constipation. A control group that was not age-matched was used on purpose, because we needed to be certain that the controls did not have the disease. None of the subjects were related to any other subjects in the study.

\section{Selection of candidate genes and SNPs}

The two candidate genes, RET-protooncogene and NRG1, were chosen based on the data from the GWAS referred to earlier, ${ }^{7}$ and our own previous study. ${ }^{5}$ The haplotype tagging SNPs were selected from the International HapMap project database, based on criteria of $r^{2}$ more than 0.8 and minor allele frequency more than 0.05 on individual genotyping.

Among the $10 \mathrm{SNPs}$ in the RET-protooncogene, we included one SNP (rs2435357) that indicated a marker SNP from the published GWAS, ${ }^{7}$ one tagSNP rs2506011 in intron 1 based on the HapMap database and linkage disequilibrium (LD) information in the Chinese population, which was assumed to be closely related genetically to the Thai population. The SNP rs 2506011 was in the same LD block with rs2435357. ${ }^{7}$ To complete the fine mapping within the region where rs 2506011 belonged to, five captured SNPs of rs2506011 (rs2505540, rs2506021, rs2506020, rs2506010 and rs3123655) were picked. In addition, three SNPs from our previous study ${ }^{5}$ (rs1800858, rs1800861, rs1800862) were included for re-validation.

Regarding the NRG1 SNPs, rs16879552 and rs7835688 were selected from the GWAS data. ${ }^{7}$ We also included the tagSNP rs2439305, which was in the same LD block as rs16879552 and rs7835688, according to the HapMap database. The SNPs were located within the suspected HSCR-susceptible region in intron 1. As this study was considered an early association study on NRG1 and HSCR, we did not focus on a complete fine mapping for the intron 1 of NRG1, and captured SNPs were not included. Additionally, we decided to explore the rs2919381, a non-coding SNP in intron 5, which was predicted to be an intronic enhancer by the FASTSNP program, ${ }^{17}$ which is a program that prioritizes SNPs based on their functional effects. The rs2919381 was among the non-coding SNPs that had high-risk scores and were located some distance away from the first group.

\section{SNP genotyping}

Genomic DNA extraction. Genomic DNA was extracted from frozen colonic tissues taken from the 68 HSCR cases, and from peripheral blood leukocytes of the 120 control samples, using the QIAamp DNA Mini Kit (QIAGEN, Hilden, Germany)

TaqMan SNP genotyping assays. Thirteen SNPs from the two genes were genotyped using custom TaqMan SNP Genotyping Assays on the 7900HT Fast Real-Time PCR system (Applied Biosystems, Foster City, CA, USA). The
TaqMan probes were VIC- and FAM-dye-labeled. The PCR used $10 \mathrm{ng}$ of genomic DNA in a total reaction volume of $5 \mu \mathrm{l}$ that consisted of TaqMan universal master mix (Applied Biosystems) $2.5 \mu \mathrm{l}$ and a $40 \times$ primer-probe mixture $0.125 \mu$ l. Mock (no template) controls were included in each run. Each reaction was amplified at $95^{\circ} \mathrm{C}$ for $10 \mathrm{~min}$, followed by 40 cycles of $95^{\circ} \mathrm{C}$ for $15 \mathrm{~s}$ and $60^{\circ} \mathrm{C}$ for $1 \mathrm{~min}$.

\section{PCR-restriction fragment length polymorphism}

To genotype the c135 G/A polymorphisms of the SNP rs1800858, PCR amplification of exon 2 of the RET-protooncogene was performed as presented in our previous study. ${ }^{5}$ Briefly, the $390 \mathrm{bp}$ amplified fragment was cut using the BstZI enzyme (Promega, Fitchburg, WI, USA) according to the manufacturer's instructions. The digested products were analyzed on 3\% agarose gel, in which individuals with GG (major allele homozygosity) showed two bands of 107 and $283 \mathrm{bp}$, those with GA (heterozygosity) showed three bands of 107, 283 and $390 \mathrm{bp}$, and those with AA (minor allele homozygosity) showed a single undigested band of $390 \mathrm{bp}$ (not shown).

\section{Genotyping quality control}

We compared genotype call rates and concordances among each individual study and the overall study. We used the following criteria as a measure of acceptable genotyping: (1) >10\% sample duplicates included; (2) concordance rate for the duplicates $\geqslant 98 \%$; (3) overall call rate by study $>95 \%$, and (4) call rates $>90 \%$ for each individual 384-well plate. The data for any SNP failing these criteria in any assays were excluded from the final analyses. The HardyWeinberg Equilibrium was checked for all SNPs $(P>0.05)$.

Available oligonucleotide sequences and the genotyping method used with each SNP are shown in Table 1 according to their genomic context. The GenBank accession numbers of the RET-protooncogene and NRG1 reference sequences used in this study were NT_033985.6 and NT_007995.14, respectively.

\section{Statistical analysis}

For statistical analysis of the SNP association with the disease, both overall analysis and subgroup analysis (Thai-Chinese and Thai-Muslim), we used Fisher's exact test with a two-by-two contingency table under allelic, dominant and recessive inheritance models. $P$-values were two-tailed and a value of less than 0.05 was considered statistically significant. Correction for multiple parameters used a conservative Bonferroni correction for the 14 SNPs studied $(P<0.05 / 14$ SNPs $=0.0036)$. Odds ratios $(\mathrm{OR})$ and $95 \%$ confidence intervals $(\mathrm{CI})$ were calculated on the risk genotypes.

The pairwise LD tests and haplotype analyses were performed with the Haploview software (www.broad.mit.edu/personal/jcbarret/haploview).

The SNP-SNP interaction analysis was conducted on the two genes to construct a combined allelotype that was associated with the disease. To examine whether marker combination may possess better predictability than individual marker alone, based on the parameters such as the most significance level, OR and frequency of the SNP allele were selected for disease marker. We selected rs2435357 (RET-protooncogene) and rs2439305 (NRG1) for the SNPSNP interaction analysis, as the two genes that showed the most significant disease association. For gene-gene interactions, the multiplicative interaction effect of the SNPs was estimated using a multiple logistic regression model. For each individual, key variables were defined as a binary variable indicating casecontrol status, with SNP variable ranging from 0 to 2 , indicating the number of risk alleles in an individual subject, according to the method described by Lewis. ${ }^{18}$

\section{RESULTS}

\section{Characteristics of the study population}

Of the 68 Thai patients with sporadic HSCR included in the study, there were 50 patients with short-segment HSCR (73.53\%), 14 patients with long-segment HSCR (20.59\%) and four patients with total colonic aganglionosis $(5.88 \%)$. There were two patients with Down's syndrome (2.94\%). The male to female ratio was 59:9 or 6.6:1.

Table 2 compares sex, age, and race between the cases and the control group. There were significant differences in sex and age, but 
Table 1 Oligonucleotide sequences and methods for genotyping of the 14 study SNPs

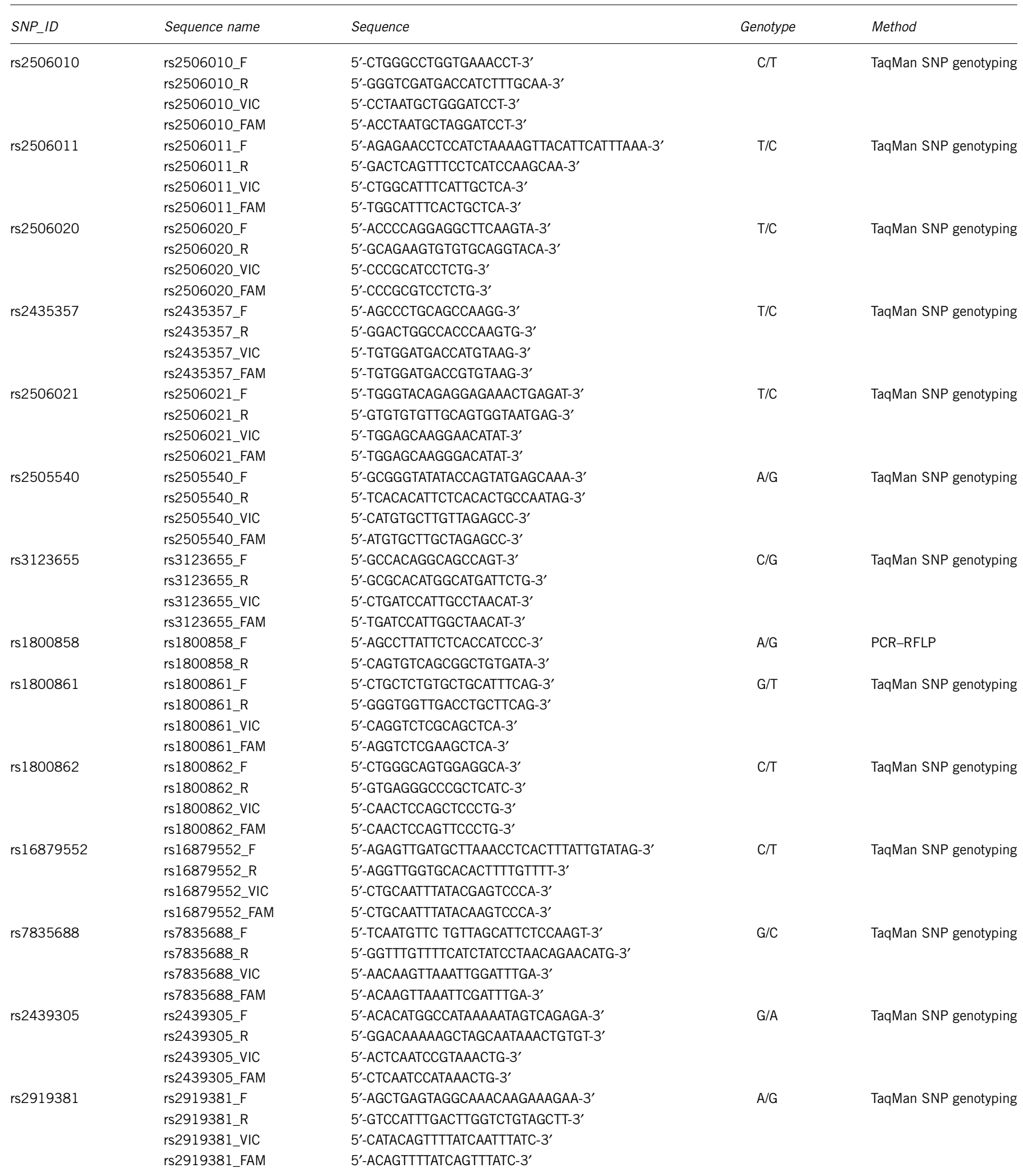

Abbreviations: RFLP, restriction fragment length polymorphism; SNP, single-nucleotide polymorphism. 
other parameters including ethnic group and severity of the disease were comparable between the two groups. There was no statistically significant difference in genotype distribution between the two ethnic

Table 2 Demographic characteristics of subjects

\begin{tabular}{lccc}
\hline Characteristic & Cases & Controls & P-value \\
\hline $\begin{array}{l}\text { No of subjects } \\
\text { Age+s.d. (years) }\end{array}$ & 68 & 120 & - \\
Sex (no.) & $1.2 \pm 2.1^{\text {a }}$ & $34.5 \pm 10.3^{\mathrm{a}}$ & 0.0001 \\
$\quad$ Female & 9 & & \\
Male & 59 & 55 & $<0.0001$ \\
& & 65 & - \\
Ethnic groups & & & 0.21 \\
Thai-Chinese & 38 & 79 & - \\
Thai-Muslim & 30 & 41 &
\end{tabular}

groups studied. Also, the genotype distribution had no significant difference between sexes.

Association between the SNPs in the RET-protooncogene and HSCR A total of 10 SNPs within the RET-protooncogene were genotyped in 68 cases and 120 controls. Of these, nine SNPs were successfully genotyped by the TaqMan SNP genotyping assay technique, and the remaining one (rs1800858) was studied by PCR-restriction fragment length polymorphism. The results of allelic and genotyping associations in the RET-protooncogene are summarized in Table 3. Significant associations between seven haplotype tagging SNPs in intron 1 of the RET-protooncogene and HSCR were demonstrated in our study. As shown in Table 3 , the most significant $P$-value was $8.17 \mathrm{E}-09$ with an OR of 6.43 (95\% CI 3.33-12.40). The allele, genotype and haplotype block identified by seven SNPs (rs2506010, rs2506011, rs2506020, rs2435357, rs2506021, rs2505540 and rs3123655), spanning an area of 23 -kb core-risk haplotype surrounding intron 1 of the gene, are strongly associated with the disease as shown in Figure 1. Pairwise LD for all possible pairs of SNPs was calculated for cases and controls.

Table 3 Summary results of the association between RET-protooncogene and HSCR

\begin{tabular}{|c|c|c|c|c|c|c|c|c|c|c|c|c|c|}
\hline \multirow[b]{3}{*}{$S N P_{-} I D$} & \multirow[b]{3}{*}{ Position $^{\mathrm{a}}$} & \multirow[b]{3}{*}{ Allele (1/2) } & \multicolumn{4}{|c|}{ Case } & \multicolumn{4}{|c|}{ Control } & \multirow[b]{3}{*}{ Risk allele } & \multirow[b]{3}{*}{ P-value ${ }^{b}$} & \multirow[b]{3}{*}{ OR $(95 \% \mathrm{Cl})$} \\
\hline & & & \multicolumn{4}{|c|}{ Genotype } & \multicolumn{4}{|c|}{ Genotype } & & & \\
\hline & & & 11 & 12 & 22 & $M A F$ & 11 & 12 & 22 & $M A F$ & & & \\
\hline \multirow[t]{3}{*}{ rs2506010 } & 42893564 (intron 1) & $\mathrm{C} / \mathrm{T}$ & 2 & 5 & 31 & 0.118 & 7 & 42 & 30 & 0.354 & $\mathrm{~T}$ & $9.91 \mathrm{E}-06^{\mathrm{c}}$ & $7.23(2.83-18.47)^{\mathrm{c}}$ \\
\hline & & & 1 & 6 & 23 & 0.133 & 2 & 17 & 22 & 0.256 & $\mathrm{~T}$ & $7.99 \mathrm{E}-02^{\mathrm{d}}$ & $2.84(1.00-8.07)^{d}$ \\
\hline & & & 3 & 11 & 54 & 0.125 & 9 & 59 & 52 & 0.321 & $\mathrm{~T}$ & $1.57 \mathrm{E}-06^{\mathrm{e}}$ & $5.04(2.53-10.05)^{\mathrm{e}}$ \\
\hline \multirow[t]{3}{*}{ rs2506011 } & 42894942 (intron 1) & $\mathrm{C} / \mathrm{T}$ & 2 & 5 & 31 & 0.118 & 7 & 42 & 30 & 0.354 & $\mathrm{~T}$ & $9.91 \mathrm{E}-06^{\mathrm{C}}$ & $7.23(2.83-18.47)^{\mathrm{c}}$ \\
\hline & & & 1 & 6 & 23 & 0.133 & 2 & 17 & 22 & 0.256 & $\mathrm{~T}$ & $7.99 \mathrm{E}-02^{\mathrm{d}}$ & $2.84(1.00-8.07)^{d}$ \\
\hline & & & 3 & 11 & 54 & 0.125 & 9 & 59 & 52 & 0.321 & $\mathrm{~T}$ & $1.57 \mathrm{E}-06^{\mathrm{e}}$ & $5.04(2.53-10.05)^{\mathrm{e}}$ \\
\hline \multirow[t]{3}{*}{ rs2506020 } & 42899079 (intron 1) & $\mathrm{C} / \mathrm{T}$ & 2 & 5 & 31 & 0.118 & 7 & 42 & 30 & 0.354 & $\mathrm{~T}$ & $9.91 \mathrm{E}-06^{\mathrm{C}}$ & $7.23(2.83-18.47)^{\mathrm{c}}$ \\
\hline & & & 1 & 6 & 23 & 0.133 & 2 & 17 & 22 & 0.256 & $\mathrm{~T}$ & $7.99 \mathrm{E}-02^{\mathrm{d}}$ & $2.84(1.00-8.07)^{d}$ \\
\hline & & & 3 & 11 & 54 & 0.125 & 9 & 59 & 52 & 0.321 & $\mathrm{~T}$ & $1.57 \mathrm{E}-06^{\mathrm{e}}$ & $5.04(2.53-10.05)^{\mathrm{e}}$ \\
\hline \multirow[t]{3}{*}{ rs2435357 } & 42902062 (intron 1) & $\mathrm{C} / \mathrm{T}$ & 2 & 9 & 27 & 0.171 & 19 & 43 & 17 & 0.513 & $\mathrm{~T}$ & $3.26 \mathrm{E}-07^{\mathrm{c}}$ & $8.95(3.70-21.64)^{c}$ \\
\hline & & & 5 & 5 & 20 & 0.250 & 6 & 21 & 14 & 0.402 & $\mathrm{~T}$ & 8.69E-03 & $3.86(1.42-10.45)^{\mathrm{d}}$ \\
\hline & & & 7 & 14 & 47 & 0.206 & 25 & 64 & 31 & 0.475 & $\mathrm{~T}$ & 8.17E-09e & $6.43(3.33-12.40)^{\mathrm{e}}$ \\
\hline \multirow[t]{3}{*}{ rs2506021 } & 42904154 (intron 1) & $\mathrm{C} / \mathrm{T}$ & 2 & 5 & 31 & 0.118 & 7 & 42 & 30 & 0.354 & $\mathrm{~T}$ & $9.91 \mathrm{E}-06^{\mathrm{c}}$ & $7.23(2.83-18.47)^{\mathrm{c}}$ \\
\hline & & & 1 & 6 & 23 & 0.133 & 2 & 17 & 22 & 0.256 & $\mathrm{~T}$ & $7.99 \mathrm{E}-02^{\mathrm{d}}$ & $2.84(1.00-8.07)^{\mathrm{d}}$ \\
\hline & & & 3 & 11 & 54 & 0.125 & 9 & 59 & 52 & 0.321 & $\mathrm{~T}$ & $1.57 \mathrm{E}-06^{\mathrm{e}}$ & $5.04(2.53-10.05)^{e}$ \\
\hline \multirow[t]{3}{*}{ rs2505540 } & 42910246 (intron 1) & $\mathrm{G} / \mathrm{A}$ & 2 & 5 & 31 & 0.118 & 7 & 42 & 30 & 0.354 & $A$ & $9.91 \mathrm{E}-06^{\mathrm{c}}$ & $7.23(2.83-18.47)^{c}$ \\
\hline & & & 1 & 6 & 23 & 0.133 & 2 & 17 & 22 & 0.256 & $A$ & $7.99 \mathrm{E}-02^{\mathrm{d}}$ & $2.84(1.00-8.07)^{\mathrm{d}}$ \\
\hline & & & 3 & 11 & 54 & 0.125 & 9 & 59 & 52 & 0.321 & $A$ & $1.57 \mathrm{E}-06^{\mathrm{e}}$ & $5.04(2.53-10.05)^{e}$ \\
\hline \multirow[t]{3}{*}{ rs3123655 } & 42915200 (intron 1) & $\mathrm{G} / \mathrm{C}$ & 2 & 5 & 31 & 0.118 & 7 & 42 & 30 & 0.354 & $\mathrm{C}$ & $9.91 \mathrm{E}-06^{\mathrm{c}}$ & $7.23(2.83-18.47)^{\mathrm{c}}$ \\
\hline & & & 1 & 6 & 23 & 0.133 & 2 & 17 & 22 & 0.256 & C & $7.99 \mathrm{E}-02^{\mathrm{d}}$ & $2.84(1.00-8.07)^{d}$ \\
\hline & & & 3 & 11 & 54 & 0.125 & 9 & 59 & 52 & 0.321 & C & $1.57 \mathrm{E}-06^{\mathrm{e}}$ & $5.04(2.53-10.05)^{e}$ \\
\hline \multirow[t]{3}{*}{ rs1800858 } & 42915974 (exon 2) & $\mathrm{G} / \mathrm{A}$ & 22 & 13 & 3 & 0.250 & 31 & 30 & 18 & 0.418 & G & $1.38 \mathrm{E}-02^{\mathrm{c}}$ & $2.13(0.97-4.68)^{c}$ \\
\hline & & & 14 & 10 & 6 & 0.367 & 9 & 21 & 11 & 0.476 & G & $4.01 \mathrm{E}-02^{\mathrm{d}}$ & $3.11(1.11-8.72)^{d}$ \\
\hline & & & 36 & 23 & 9 & 0.301 & 40 & 51 & 29 & 0.454 & G & $4.32 \mathrm{E}-03^{e}$ & $2.25(1.22-4.14)^{\mathrm{e}}$ \\
\hline \multirow[t]{3}{*}{ rs1800861 } & 42933849 (exon 13) & $\mathrm{T} / \mathrm{G}$ & 4 & 10 & 24 & 0.237 & 19 & 41 & 19 & 0.499 & $\mathrm{G}$ & $7.42 \mathrm{E}-05^{\mathrm{C}}$ & $5.41(2.34-12.50)^{c}$ \\
\hline & & & 3 & 11 & 16 & 0.283 & 7 & 17 & 17 & 0.378 & $\mathrm{G}$ & $2.83 \mathrm{E}-01^{\mathrm{d}}$ & $1.61(0.62-4.17)^{d}$ \\
\hline & & & 7 & 21 & 40 & 0.257 & 26 & 58 & 36 & 0.458 & $\mathrm{G}$ & $1.13 \mathrm{E}-04^{\mathrm{e}}$ & $3.33(1.79-6.20)^{\mathrm{e}}$ \\
\hline \multirow[t]{3}{*}{ rs1800862 } & 42935100 (exon 14) & $\mathrm{T} / \mathrm{C}$ & 0 & 0 & 38 & 0 & 0 & 1 & 78 & 0.006 & C & $1.00 \mathrm{E}+00^{\mathrm{c}}$ & $N A^{c}$ \\
\hline & & & 0 & 1 & 28 & 0.017 & 0 & 2 & 39 & 0.024 & C & $1.00 \mathrm{E}+00^{\mathrm{d}}$ & $1.44(0.12-16.62)^{\mathrm{d}}$ \\
\hline & & & 0 & 1 & 66 & 0.007 & 0 & 3 & 117 & 0.013 & C & $1.00 \mathrm{E}+00^{\mathrm{e}}$ & $1.69(0.17-16.60)^{\mathrm{e}}$ \\
\hline
\end{tabular}

Abbreviations: $\mathrm{Cl}$, confidence interval, $\mathrm{HSCR}$, Hirschsprung disease; MAF, minor allele frequency; OR, odds ratio; SNP, single-nucleotide polymorphism.

bThe minimum $P$-values of Fisher's exact test were calculated by Fisher's exact test to compare the allelic and genotypic frequencies between cases and controls in three different models.

'Statistical analysis for the Thai-Chinese subjects.

dStatistical analysis for the Thai-Muslim subjects.

eStatistical analysis for all subjects. 

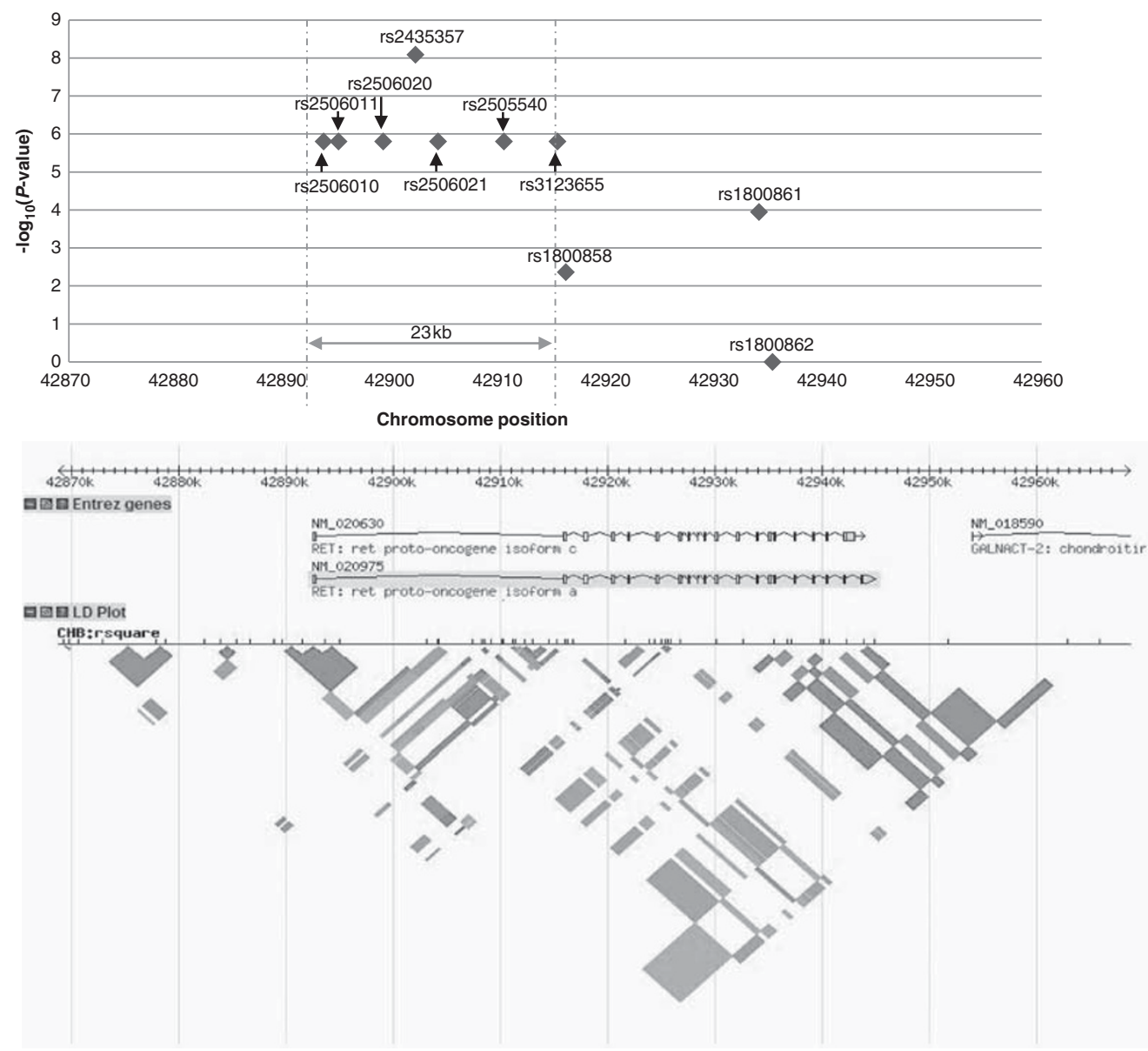

Figure 1 Case-control association $P$-value plots, LD map and genomic structure of the RET-protooncogene region on chromosome 10q11.2, spanning an area of 23-kb core-risk haplotype surrounding intron 1 of the gene. Pairwise LD $\left(r^{2}\right)$ is based on the genotype data of Han Chinese in the HapMap database. A full color version of this figure is available at the Journal of Human Genetics journal online.

Table 4 Summary results for associations between the NRG1 genotypes and HSCR

\begin{tabular}{|c|c|c|c|c|c|c|c|c|c|c|c|c|c|}
\hline \multirow[b]{3}{*}{$S N P \_I D$} & \multirow[b]{3}{*}{ Position ${ }^{\mathrm{a}}$} & \multirow[b]{3}{*}{ Allele (1/2) } & \multicolumn{4}{|c|}{ Case } & \multicolumn{4}{|c|}{ Control } & \multirow[b]{3}{*}{ Risk allele } & \multirow[b]{3}{*}{$\mathrm{P}$-value ${ }^{\mathrm{b}}$} & \multirow[b]{3}{*}{ OR $(95 \% \mathrm{Cl})$} \\
\hline & & & \multicolumn{4}{|c|}{ Genotype } & \multicolumn{4}{|c|}{ Genotype } & & & \\
\hline & & & 11 & 12 & 22 & $M A F$ & 11 & 12 & 22 & $M A F$ & & & \\
\hline \multirow[t]{3}{*}{ rs16879552 } & 32530758 (intron 1) & $\mathrm{T} / \mathrm{C}$ & 0 & 13 & 25 & 0.171 & 15 & 37 & 26 & 0.429 & C & $1.11 \mathrm{E}-04^{\mathrm{c}}$ & $3.85(1.70-8.72)^{c}$ \\
\hline & & & 5 & 7 & 18 & 0.283 & 5 & 8 & 28 & 0.219 & C & 4.33E-01d & $0.70(0.26-1.86)^{d}$ \\
\hline & & & 5 & 20 & 43 & 0.221 & 20 & 45 & 54 & 0.357 & C & $7.23 \mathrm{E}-03^{\mathrm{e}}$ & $2.07(1.12-3.81)^{\mathrm{e}}$ \\
\hline \multirow[t]{3}{*}{ rs7835688 } & 32531041 (intron 1) & $\mathrm{C} / \mathrm{G}$ & 7 & 16 & 15 & 0.395 & 6 & 27 & 45 & 0.250 & C & $3.18 \mathrm{E}-02^{\mathrm{c}}$ & $2.71(0.84-8.72)^{c}$ \\
\hline & & & 6 & 10 & 14 & 0.367 & 2 & 16 & 23 & 0.244 & C & $6.31 \mathrm{E}-02^{\mathrm{d}}$ & $4.88(0.91-26.14)^{d}$ \\
\hline & & & 13 & 26 & 29 & 0.382 & 8 & 43 & 68 & 0.248 & C & $6.94 \mathrm{E}-03^{\mathrm{e}}$ & $3.28(1.28-8.38)^{\mathrm{e}}$ \\
\hline \multirow[t]{3}{*}{ rs2439305 } & 32549006 (intron 1) & $A / G$ & 15 & 16 & 7 & 0.395 & 45 & 27 & 6 & 0.250 & G & $3.18 \mathrm{E}-02^{\mathrm{c}}$ & $2.71(0.84-8.72)^{c}$ \\
\hline & & & 14 & 10 & 6 & 0.367 & 23 & 16 & 2 & 0.244 & $\mathrm{G}$ & $6.31 \mathrm{E}-02^{\mathrm{d}}$ & $4.88(0.91-26.14)^{d}$ \\
\hline & & & 29 & 26 & 13 & 0.382 & 68 & 43 & 8 & 0.248 & G & $6.94 \mathrm{E}-03^{\mathrm{e}}$ & $3.28(1.28-8.38)^{e}$ \\
\hline \multirow[t]{3}{*}{ rs2919381 } & 32683466 (intron 5) & $\mathrm{G} / \mathrm{A}$ & 16 & 20 & 2 & 0.316 & 22 & 42 & 15 & 0.456 & G & $4.74 \mathrm{E}-02^{\mathrm{c}}$ & $1.88(0.84-4.24)^{c}$ \\
\hline & & & 16 & 10 & 4 & 0.300 & 16 & 18 & 7 & 0.390 & G & $2.90 \mathrm{E}-01^{\mathrm{d}}$ & $1.79(0.69-4.63)^{d}$ \\
\hline & & & 32 & 30 & 6 & 0.309 & 38 & 60 & 22 & 0.433 & G & $2.07 \mathrm{E}-02^{\mathrm{e}}$ & $1.92(1.04-3.54)^{\mathrm{e}}$ \\
\hline
\end{tabular}

Abbreviations: $\mathrm{Cl}$, confidence interval, HSCR, Hirschsprung disease; MAF, minor allele frequency; OR, odds ratio; SNP, single-nucleotide polymorphism.

Statistically significant associations after application of the Bonferroni correction are shown in bold, based on 14 independent effective tests $(P<0.0036)$.

aPositions of the SNPs were derived from NCBI build36.3_NT007995.14.

bThe minimum $P$-values of Fisher's exact test were calculated by Fisher's exact test to compare the allelic and genotypic frequencies between cases and controls in three different models.

cStatistical analysis for the Thai-Chinese subjects.

dStatistical analysis for the Thai-Muslim subjects.

eStatistical analysis for all subjects. 
We found a strong $\mathrm{LD}\left(D^{\prime}>0.94\right)$ in all seven non-coding region SNPs. Haplotype analysis showed that these seven risk-associated alleles presented in the same risk haplotype (TTTTTAC) of all subjects; in both the Thai-Chinese and Thai-Muslim subgroups with a $P$-value $=8.63 \mathrm{E}-08 \quad(\mathrm{OR}=3.64,95 \% \mathrm{CI}=2.24-5.92)$, a $P$-value $=$ 2.05E-07 $(\mathrm{OR}=5.44,95 \% \mathrm{CI}=2.77-10.67)$, and a $P$-value $=7.28 \mathrm{E}-02$ $(\mathrm{OR}=2.02,95 \% \mathrm{CI}=0.97-4.20)$, respectively (Supplementary Table $\mathrm{S} 1$ ).

\section{Association between the SNPs in NRG1 and HSCR}

All four SNPs were successfully genotyped by the TaqMan SNP genotyping assay. The results of association analysis for SNPs of the NRG1 and HSCR are summarized in Table 4. All SNPs studied had significant disease association; however, this association was shown to be marginal when the Bonferroni correction for multiple markers was performed (Figure 2). A pairwise LD test demonstrated that that three non-coding region SNPs in NRG1 (rs16879552, rs7835688 and rs2439305) showed a strong LD $\left(D^{\prime}=1\right)$. Haplotype analysis showed significant associations between the risk alleles (CCG) and all HSCRs at a $P$-value of $6.72 \mathrm{E}-03(\mathrm{OR}=1.90,95 \% \mathrm{CI}=1.21-2.99)$. When ethnic subgroup analysis was performed, the NRG1 haplotype showed disease association in the Thai-Chinese patients at a $P$-value of $2.25 \mathrm{E}-02$ $(\mathrm{OR}=1.99,95 \% \mathrm{CI}=1.11-3.57)$, but not in Thai-Muslim with $P$-value $=1.37 \mathrm{E}-01(\mathrm{OR}=1.79,95 \% \mathrm{CI}=0.87-3.72)$.

SNP-SNP interaction of the network that governs the development of the enteric nervous system-related genes

The rs2435357 (RET-protooncogene) and rs2439305 (NRG1) were identified as the most significant markers associated with HSCR in our cohort. Analysis of the combined genotypes of these two genes revealed a significant increase in HSCR risk with increasing numbers

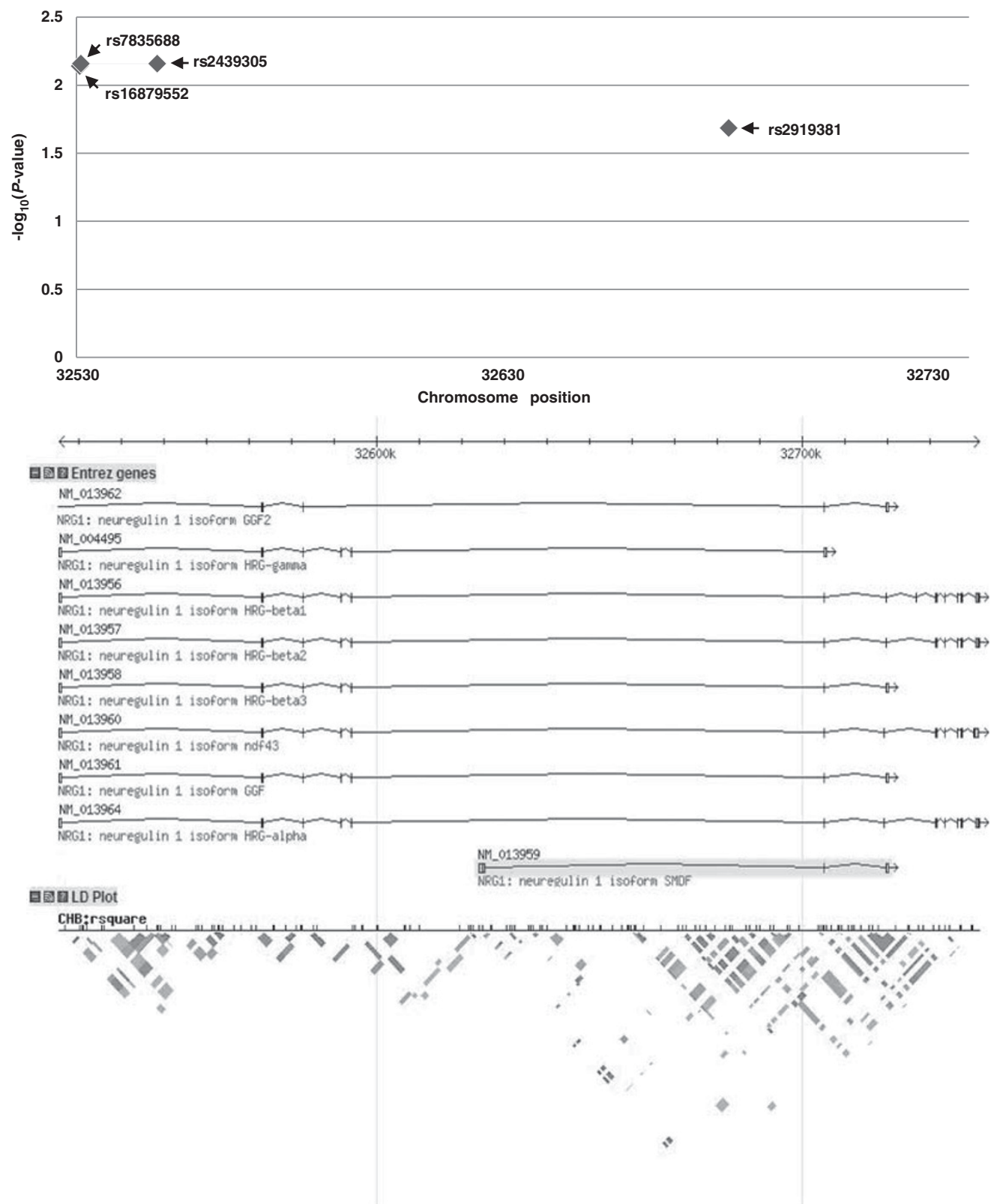

Figure 2 Case-control association P-value plots, LD map and genomic structure of the NRG1 region on chromosome 8p12. Pairwise LD $\left(r^{2}\right)$ was based on the genotype data of Han Chinese in HapMap database. A full color version of this figure is available at the Journal of Human Genetics journal online. 
Table 5 OR and corresponding $95 \% \mathrm{Cl}$ for the joint effects of SNP-SNP interaction and HSCR risk

\begin{tabular}{|c|c|c|c|c|c|c|c|}
\hline \multicolumn{2}{|c|}{ SNP-SNP interaction } & \multicolumn{2}{|c|}{ Combination } & \multicolumn{2}{|c|}{ Frequency $\mathrm{N},(\%)$} & \multirow[b]{2}{*}{ OR ratio $(95 \% \mathrm{Cl})$} & \multirow[b]{2}{*}{ P-value ${ }^{a}$} \\
\hline RET & $N R G 1$ & Genotype & Genotype & Controls & Patients & & \\
\hline \multirow[t]{7}{*}{ rs2435357 } & rs2439305 & $\mathrm{CC}$ & $A A$ & $14(11.76)$ & $6(8.82)$ & Reference & \\
\hline & & - & $A G$ & $10(8.40)$ & $1(1.47)$ & $0.16(0.02-1.30)$ & $1.01 \mathrm{E}-01$ \\
\hline & & - & $\mathrm{GG}$ & $1(0.84)$ & $0(0)$ & NA & $1.00 \mathrm{E}+00$ \\
\hline & & - & GG & $6(5.04)$ & $3(4.41)$ & $0.87(0.21-3.59)$ & $1.00 \mathrm{E}+00$ \\
\hline & & $\mathrm{TT}$ & $A A$ & $18(15.13)$ & $19(27.94)$ & $2.18(1.05-4.51)$ & $3.80 \mathrm{E}-02$ \\
\hline & & - & $A G$ & $12(10.08)$ & $18(26.47)$ & $3.21(1.44-7.17)$ & $4.15 \mathrm{E}-03$ \\
\hline & & - & GG & $1(0.84)$ & $10(14.71)$ & $20.34(2.54-162.78)$ & $1.99 \mathrm{E}-04$ \\
\hline
\end{tabular}

Abbreviations: $\mathrm{Cl}$, confidence interval, HSCR, Hirschsprung disease; N, number of subjects; OR, odds ratio; SNP, single-nucleotide polymorphism.

aMultiple logistic regression comparison.

of putative high-risk alleles. Table 5 presents the SNP-SNP interaction results in all models. Under a recessive model, the risk of HSCR was highest in those subjects harboring homozygous risk alleles (TT) of rs2435357 and homozygous risk alleles (GG) of rs2439305 $(\mathrm{OR}=20.34,95 \% \mathrm{CI}=2.54-162.78)$, compared with other genotypes. There was no or minimal increased risk for HSCR at these two loci if only one risk allele was present: $\mathrm{OR}=0.74(95 \% \mathrm{CI}=0.18-2.95)$ for the presence of the NRG1 risk allele but not the RET-protooncogene risk allele, and $\mathrm{OR}=3.54(95 \% \mathrm{CI}=1.88-6.66)$ for the presence of the RET-protooncogene risk allele but not the NRG1 risk allele.

\section{DISCUSSION}

In this study, two candidate genes that are known to be involved with control of the development of the enteric nervous system, RETprotooncogene and $N R G 1$, were simultaneously examined using a real-time PCR-based genotyping technique. Using the model of genetic susceptibility in multifactorial disease, we identified disease association of the genetic variants in RET-protooncogene and NRG1 with sporadic HSCR in Thai patients.

The RET-protooncogene, located on chromosome 10, is composed of 21 exons and encodes a $52.42 \mathrm{~kb}$ size tyrosine kinase receptor involved in growth, migration and differentiation of enteric neurons. ${ }^{19}$ According to GWAS data, ${ }^{7}$ rs 2435357 represents a marker for an LD locus in the RET-protooncogene that needs further investigation. Functionally, rs 2435357 has been proven to lie in the enhancer-like sequence within intron 1 of the RET-protooncogene. ${ }^{20}$ To conduct a fine-mapping study for the most significant genetic variants associated with HSCR in the Thai population, more haplotype tagging SNPs covering intron 1 of the RET-protooncogene were included in our analysis. We further genotyped for rs1800858, rs1800861 and rs1800862, which have been reported to have disease association in previous studies. ${ }^{21,22}$ Using a high-throughput genotyping technique, our case-control study successfully validated an association between HSCR in the Thai population and the SNP within the LD block of the RET-protooncogene with a high OR.

$N R G 1$, a 1.1-Mb gene located on chromosome $8 \mathrm{p} 12$, belongs to a family of structurally related glycoproteins that act as multifunctional factors that bind to ErbB receptors and are involved in neuronal cell differentiation. ${ }^{23-26}$ A HapMap database constructed from a GWAS has suggested a statistically significant association between the SNPs under the LD block in NRG1 and HSCR. ${ }^{7}$ A recent validation study in the Chinese population also confirmed this disease association.
Focusing on the SNPs within NRG1, our study found an association between NRG1 polymorphisms and HSCR in Thais at a comparable OR with the Chinese study noted above. ${ }^{7}$

We further evaluated the interaction between the RET-protooncogene and NRG1 by analyzing the combination of landmark SNPs from the two genes for any disease association. Our main finding was that genetic interactions significantly modulated the risk for HSCR in our Thai casecontrol subjects. Interestingly, the combination between polymorphisms of RET-protooncogene and NRG1 was associated with a 20-fold increased risk of the disease in individuals who carried the risk alleles of these polymorphisms compared with individuals whose genotype contained neither risk allele. These results are in line with a previous study that demonstrated interactions between rs2435357 in RET-protooncogene, and the rs16879552 and rs7835688 in the NRG1 in the Chinese population, ${ }^{7}$ in the way that the two NRG1 SNPs reported in their study were in high LD with the rs2439305 SNP reported in this study. $N R G 1$ is involved in neuronal development by promoting neuronal survival, whereas the RET-protooncogene may perform proliferative and/ or main differentiation functions. ${ }^{8,25}$ The mechanism of interaction between the two genes is still not well understood, because very few studies have considered such interactions, and therefore, it would be pure speculation to try to explain the interactions found in this study on the basis of known functions. However, epidemiological analyses of interacting variants will lead to a better understanding of the biological pathways underlying HSCR. Considering a relatively high specificity, a SNP or a combination of SNPs can be used as a disease marker together with clinical parameters for screening for HSCR in individual cases. Further prospective validation studies with a larger number of participants are required to confirm the predictive power of the markers.

Our study found that real-time PCR with Taqman probes is a rapid and simple method for SNP genotyping. To our knowledge, this is the first study that has used a fluorescence-based SNP detection assay for genotyping RET-protooncogene and NRG1, as previous studies have usually used PCR-direct sequencing, single-stranded conformation polymorphisms and restriction fragment length polymorphisms. ${ }^{5,13,14,16,20,21}$ This fluorescence-based technique relies on different properties of two probes that are specific to the alleles of interest, each labeled with different fluorescent markers. It offers several advantages compared with older techniques; not only does it feature high specificity and accuracy, but also not requiring a post-amplification step reduces the time and cost of genotyping. In this study, only one of the SNP (rs1800858) failed to use this method. 
In conclusion, we identified SNPs in RET-protooncogene and NRG1, which were disease-associated with Thai HSCR. We also demonstrated epidemiological interaction between the two genes.

\section{CONFLICT OF INTEREST}

The authors declare no conflict of interest.

\section{ACKNOWLEDGEMENTS}

The study was supported by grant from under the program Strategic Scholarships for Frontier Research Network for the Ph.D. Program Thai Doctoral degree from the Office of the Higher Education Commission, Thailand, and the Graduate Fund of Prince of Songkla University. We thank the Blood Bank Unit of Songklanagarind and Pattani Hospitals for the control samples. Dave Patterson helped with English language editing.

Author contributions: All authors contributed to this study as declared below; T Phusantisampan: experiments, data analysis, manuscript writing; S Sangkhathat: manuscript writing, data analysis; A Phongdara: methodology review; P Chiengkriwate, S Patrapinyokul: samples collection and clinical data provision; S Mahasirimongkol: instrument provision and technical consultant.

1 Amiel, J. \& Lyonnet, S. Hirschsprung disease, associated syndromes, and genetics: a review. J. Med. Genet. 38, 729-739 (2001).

2 Badner, J. A., Sieber, W. K., Garver, K. L. \& Chakravarti, A. A genetic study of Hirschsprung disease. Am. J. Hum. Genet. 46, 568-580 (1990).

3 Bodian, M., Carter, C. O. \& Ward, B. C. Hirschsprung's disease. Lancet 1, 302-309 (1951).

4 Amiel, J., Sproat-Emison, E., Garcia-Barcelo, M., Lantieri, F., Burzynski, G., Borrego, S et al. Hirschsprung disease, associated syndromes and genetics: a review. J. Med. Genet. 45, 1-14 (2008).

5 Sangkhathat, S., Kusafuka, T., Chengkriwate, P., Patrapinyokul, S., Sangthong, B. \& Fukuzawa, M. Mutations and polymorphisms of Hirschsprung disease candidate genes in Thai patients. J. Hum. Genet. 51, 1126-1132 (2006).

6 Tam, P. K. \& Garcia-Barcelo, M. Molecular genetics of Hirschsprung's disease. Semin. Pediatr. Surg. 13, 236-248 (2004).

7 Garcia-Barcelo, M. M., Tang, C. S., Ngan, E. S., Lui, V. C., Chen, Y., So, M. T. et al. Genome-wide association study identifies NRG1 as a susceptibility locus for Hirschsprung's disease. Proc. Natl Acad. Sci. USA. 106, 2694-2699 (2009).

8 Tam, P. K. \& Garcia-Barcelo, M. M. Genetic basis of Hirschsprung's disease. Pediatr Surg. Int. 25, 543-558 (2009).

9 Iwashita, T., Kruger, G. M., Pardal, R., Kiel, M. J. \& Morrison, M. J. Hirschsprung disease is linked to defects in neural crest stem cell function. Science 301, 972-976 (2003).

10 Tam, P. K., Gould, S. J., Martucciello, G., Biddolph, S, Takahashi, M. \& Jasonni, V. Ret protein in the human fetal rectum. J. Pediatr. Surg. 31, 568-571 (1996).
11 Carrasquillo, M. M., McCallion, A. S., Puffenberger, E. G., Kashuk, C. S., Nouri, N. \& Chakravarti, A. Genome-wide association study and mouse model identify interaction between RET and EDNRB pathways in Hirschsprung disease. Nat. Genet. 32, 237-244 (2002).

12 Garcia-Barcelo, M. M., Sham, M. H., Lui, V. C., Chen, B. L., Song, Y. Q., Lee, W. S. et al. Chinese patients with sporadic Hirschsprung's disease are predominantly represented by a single RET haplotype. J. Med. Genet. 40, e122 (2002).

13 Garcia-Barcelo, M. M., Sham, M. H., Lee, W. S., Lui, V. C., Chen, B. L., Wong, K. K. et al. Highly recurrent RET mutations and novel mutations in genes of the receptor tyrosine kinase and endothelin receptor B pathways in Chinese patients with sporadic Hirschsprung disease. Clin. Chem. 50, 93-100 (2004).

14 Kim, J., Yoon, K. O., Kim, J. K., Kim, J. W., Lee, S. K., Kong, S. Y. et al. Novel mutations of RET gene in Korean patients with sporadic Hirschsprung's disease. J. Pediatr. Surg. 41, 1250-1254 (2006).

15 Kusafuka, T, Wang, Y. \& Puri, P. Mutation analysis of the RET, the endothelin-B receptor, and the endothelin-3 genes in sporadic cases of Hirschsprung's disease. J. Pediatr. Surg. 32, 501-504 (1997).

16 Wu, T. T., Tsai, T. W., Chu, C. T., Lee, Z. F., Hung, C. M., Su, C. C. et al. Low RET mutation frequency and polymorphism analysis of the RET and EDNRB genes in patients with Hirschsprung disease in Taiwan. J. Hum. Genet. 50, 168-174 (2005).

17 Yuan, H. Y., Chiou, J. J., Tseng, W. H., Liu, C. H., Liu, C. K., Lin, Y. J. et al. FASTSNP: an always up-to-date and extendable service for SNP function analysis and prioritization. Nucleic Acids Res. 34, W635-641 (2006).

18 Lewis, C. M. Genetic association studies: design, analysis and interpretation. Brief Bioinform. 3, 146-153 (2002).

19 Kusafuka, T. \& Puri, P. The RET proto-oncogene: a challenge to our understanding of disease pathogenesis. Pediatr. Surg. Int. 12, 11-18 (1997).

20 Emison, E. S., McCallion, A. S., Kashuk, C. S., Bush, R. T., Grice, E., Lin, S. A. et al. Common sex-dependent mutation in a RET enhancer underlies Hirschsprung disease risk. Nature 434, 857-863 (2005).

21 Borrego, S., Saez, M. E., Ruiz, A., Gimm, O., Lopez-Alonso, M., Antinolo, G. et al. Specific polymorphisms in the RET proto-oncogene are over-represented in patients with Hirschsprung disease and may represent loci modifying phenotypic expression. J. Med. Genet. 36, 771-774 (1999).

22 Sancandi, M., Ceccherini, I., Costa, M., Fava, M., Chen, B., Wu, Y. et al. Incidence of RET mutations in patients with Hirschsprung's disease. J. Pediatr. Surg. 35, 139-142 (2000).

23 Hancock, M. L., Canetta, S. E., Role, L. W. \& Talmage, D. A Presynaptic type III neuregulin1-ErbB signaling targets alpha7 nicotinic acetylcholine receptors to axons. J. Gen. Physiol. 131, i4 (2008).

24 Hippenmeyer, S., Shneider, N. A., Birchmeier, C., Burden, S. J., Jessell, T. M. \& Arber, S A role for neuregulin1 signaling in muscle spindle differentiation. Neuron 36, 1035-1049 (2002).

25 Krug, A., Markov, V., Eggermann, T., Krach, S., Zerres, K., Stocker, T. et al. Genetic variation in the schizophrenia-risk gene neuregulin1 correlates with differences in frontal brain activation in a working memory task in healthy individuals. Neuroimage 42, 1569-1576 (2008).

26 Sei, Y., Ren-Patterson, R., Li, Z., Tunbridge, E. M., Egan, M. F., Kolachana, B. S. et al. Neuregulin1-induced cell migration is impaired in schizophrenia: association with neuregulin1 and catechol-o-methyltransferase gene polymorphisms. Mol. Psychiatry 12, 946-957 (2007).

Supplementary Information accompanies the paper on Journal of Human Genetics website (http://www.nature.com/jhg) 